\title{
Using threshold values to continuously evaluate how effectively electrostatic precipitators operate
}

\author{
Bastian Alt ${ }^{1}$ (D) $\cdot$ Daniel Klüh ${ }^{1} \cdot$ Katharina Koch ${ }^{1} \cdot$ Bernhard Huber $^{1} \cdot$ Matthias Gaderer $^{1}$
}

Received: 2 June 2020 / Revised: 2 September 2020 / Accepted: 10 September 2020 / Published online: 18 September 2020

(C) The Author(s) 2020

\begin{abstract}
The increased use of biomass as a fuel in energy production can reduce $\mathrm{CO}_{2}$ emissions in this sector. Fossil fuels can thus be replaced, especially in the field of heat supply. However, this should not occur at the expense of increased particulate matter emissions, which is why electrostatic precipitators (ESPs) are used. Continuous monitoring of the ESP is called for in the European Union Directive 2015/2193 for medium combustion plants (MCP), which applies to plants with a nominal heat output of between 1 and $50 \mathrm{MW}$. A monitoring methodology based on the precipitator parameters current $(I)$ and voltage $(U)$ was presented in an earlier presentation. The work at hand follows up on the previous findings and presents an alternative methodology that aims to define the effectiveness of the ESP via threshold values for $U$ and $I$. It is shown that two types of misinterpretation of the operation may occur when threshold values are defined. The operating condition could be regarded as effective via the methodology, although the measurement proves ineffective in operation and the opposite error also occurs. The statistical evaluation of the frequency of errors is intended to help the legislative authorities determine the threshold values in a reasonable way. In addition, checks are made as to whether it is possible to verify the operation of the combustion system via the flue gas temperature. The operating times of the combustion are essential, as they define the operating times required by the precipitator. In this context, statistical evaluations are made to determine which plant-specific threshold value could be useful. The aim is to test the effective operation of the separator from readily available data at all times. These considerations could form the basis for developing a uniform methodology to ensure compliance with the legal requirements.
\end{abstract}

Keywords Emissions $\cdot$ Combustion $\cdot$ Flue gas cleaning $\cdot$ Electrostatic precipitators $\cdot$ Precipitation monitoring $\cdot$ Bioenergy

\section{Introduction}

The discussion in society and politics about the reduction of greenhouse gases is ongoing [1]. Since climate change is a global issue, several climate summits have been held to find a consensus on the worldwide reduction of greenhouse gases; the latest was the one held in Madrid in 2019 [2]. Existing technologies and new technological advances can contribute to $\mathrm{CO}_{2}$ reduction goals [3]. The thermal utilisation of biomass in the heating and power sector is an available technology that can cut $\mathrm{CO}_{2}$ emissions dramatically compared with using

Bastian Alt

bastian.alt@tum.de

1 Regenerative Energy Systems, Technical University of Munich, Campus Straubing for Biotechnology and Sustainability, Schulgasse 16, 94315 Straubing, Germany fossil fuels. However, the availability of sustainable biomass sources is limited [4].

The combustion of woody biomass has one major drawback: higher particulate matter concentration in the flue gas than in, for example, gas and oil [5]. This particulate matter has to be removed with secondary abatement technologies such as bag filters, cyclones or electrostatic precipitators (ESP). These technologies can lower the particulate matter emissions reliably to the emission limit defined by a regulatory framework [6, 7]. One framework is the European Union Directive 2015/2193 for medium combustion plants (MCP). This regulation specifies that the effective operation of the abatement technology has to be proven continuously. In Germany, this was implemented in 2019 in the 44th Federal Immission Control Act (44. Bundesimmissionsschutzverordnung or BImSchV) [8]. In this context, plants that have a nominal heat output of between 1 and $5 \mathrm{MW}$ can avoid the need for uninterrupted measurement by continuously proving the effective operation of the ESP when a state-of-the-art method is available [8]. A low-cost 
method is therefore needed in order to sustain the economic competitiveness of biomass combustion plants [9].

Using a measurement device will lead to additional costs that need to be avoided in order to reduce the investment and operational costs of the plant. The method proposed is based on the readily available current and voltage supplied to the electrostatic precipitator. A previous paper developed a monitoring method and showed its successful application on two biomass heating plants [10]. However, the method has to be further refined and tested to guarantee the reliability. The reliability of the method is important as it is planned to transform it into an approved standard for continuous monitoring to fulfil the regulatory framework.

In the course of the investigations, the method proposed in [10] was also tested on a plant with a modulating load. The results using the original proposal were not satisfactory because the plant did not have a stationary operation mode. For that reason, a new approach is being taken that uses threshold values for voltage and current to define the effective operation of the precipitator.Regardless of the choice of monitoring methodology, it is important to know the times at which the combustion plant is operated. The operation of the ESP must then be measured at these times. Therefore, it is discussed how the operation of the combustion system can be determined by the exhaust gas temperature. This makes the monitoring methodology independent of signals from the combustion system manufacturer.

The new approach is derived from the legislation that applies in Switzerland. The FAQ 38 of the QM Holzheizwerke also defines threshold values [11]. This approach was developed by Nussbaumer et al. by using point measurements at multiple plants [12]. In our work, the difference is that we use continuously measured data to evaluate whether the definition of threshold values actually defines an effective operation point of the precipitator. In contrast to other researchers, our goal is not to improve the ESP performance [13-16], and we do not focus on the theoretical evaluation of ESP physics [17-20] or on the numerical simulation of the ESP [21-23], but it is to use statistical evaluation in order to find a uniform monitoring method.

\section{Methods and approach}

In a previous paper [10], the efficiency calculation for the particle precipitation was presented. This uses the available current and voltage values of the high-voltage unit supply of an ESP. The method is based on the "Deutsch equation", which attempts to describe the physics of the precipitation process [24-27]. This approach was used to create Eq. 1 for calculating the precipitation efficiency.

$\eta=1-\exp (-k \cdot U \cdot I)$
The value of $k$ describes the specific precipitation constant that includes all the physical properties that can be assumed to be constant under stationary operation conditions (geometry of the electrodes, flow velocity, etc.). The aim of this method was to avoid the high costs of continuous measurements and yet still be able to validate the mentioned function. One significant result was that the deviation between measurement and calculation is less than $7 \%$ for plants that operate under stationary conditions, when the operation is comparable with the one during adjustment of the method [10].

The measurement setup consists of continuously operating particulate matter sensors using light scattering and gravimetric particulate matter measurement equipment working according to VDI 2066 part 1 for calibration [28]. Additionally, gas analysers to determine the flue gas temperature as well as flow velocity via differential pressure sensors were used $[10,29,30]$. The particle matter concentration was continuously measured before and after the ESP to determine the precipitation efficiency.

For this work, four biomass heating plants with thermal outputs of $0.75 \mathrm{MW}$ (plant 1), 1.4 MW (plant 2), 2.0 MW (plant 3) and 1.4 MW (plant 4) were evaluated. All plants use wood chips on a grate firing. Plants 1 and 3 use a tubular ESP and operate in a cyclic mode that is further explained in Section 3.4. Plants 2 and 4 are equipped with plate ESPs and are operated full time with modulating power.

The voltage and current signals are extracted from the control system of the ESP and can be used to determine the precipitator's performance. Two different approaches lead to promising results: calculating the precipitation efficiency by using Eq. 1 or defining threshold values for $U$ and $I$. This means that the precipitator is running efficiently when the present value of both signals is higher than the corresponding threshold value. The thresholds are defined as ratios of the maximum voltage or current. If the measured current is lower than $20 \%$ of the nominal current and/or the measured voltage is lower than $50 \%$ of the nominal voltage, the ESP operation is defined as inefficient. These percentage values are derived from the Swiss legislation mentioned before [11]. This monitoring method can be visualised as seen in Fig. 1, where the grey area marks the invalid operation points.

The following chapter presents the issues of the method based on Eq. 1. After that, the results of the threshold method are discussed based on how accurate this method is and which threshold values are reasonable.

The evaluation of the plants is based on defining a target value. For plants 1 and 3, a precipitation efficiency of $90 \%$ was set as target value. This was because the cyclone was built inside the housing of the ESP at these plants. Consequently, the precipitation efficiency of the cyclone was integrated [10]. At plants 2 and 4, it was possible to measure the particulate matter concentration between the cyclone and the ESP. In 
Fig. 1 Using threshold values to define the invalid operation area (grey) of the precipitator

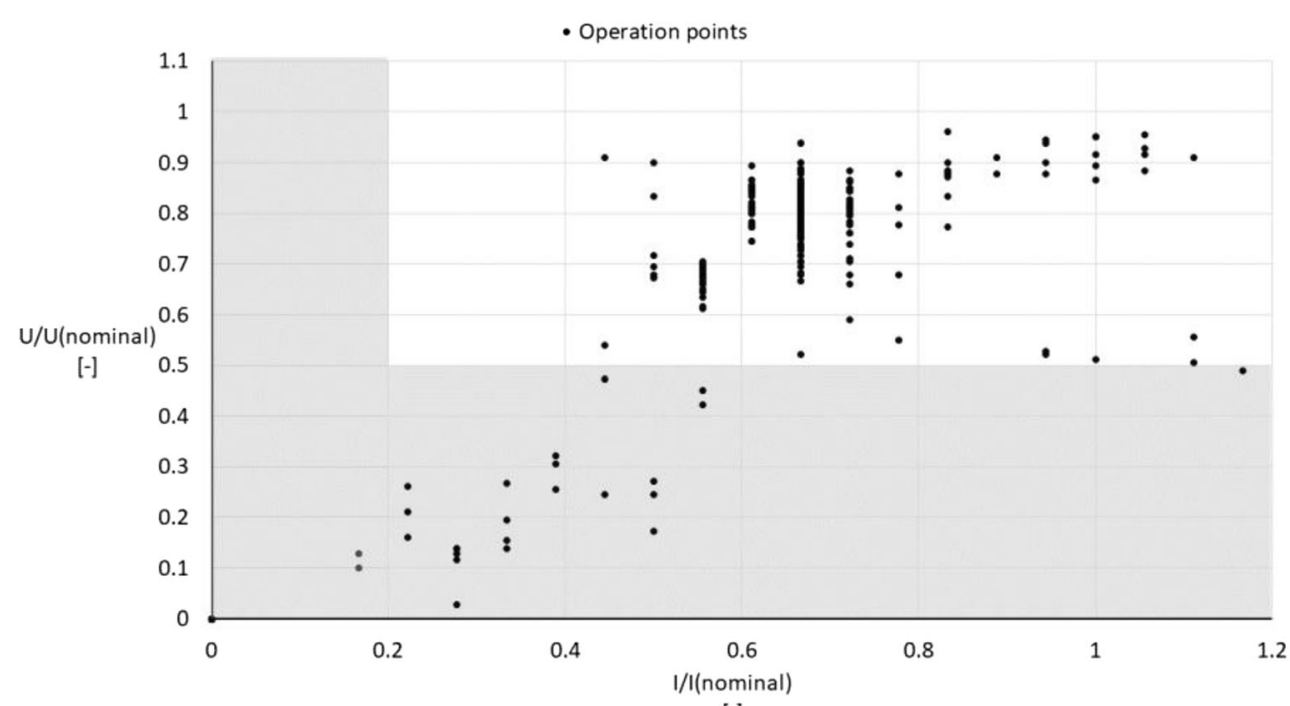

$[-]$ conclusion, only the precipitation efficiency of the electrostatic precipitator was determined. The target values were set to $60 \%$ for plant 2 and $85 \%$ for plant 4 . These values were chosen because this precipitation efficiency would directly correlate with reaching the legal emission limit values for each plant. As these plants are operated in Germany, the emissions' limits are $20 \mathrm{mg} / \mathrm{m}^{3}$ under nominal conditions referring to an oxygen concentration of $13 \%$ for plant 1 and $50 \mathrm{mg} / \mathrm{m}^{3}$ under nominal conditions referring to an oxygen concentration of $6 \%$ for the other plants.

\section{Results and discussion}

\subsection{Evaluation of the equation-based method}

An attempt to evaluate the precipitator performance by calculating precipitation efficiency had been performed previously [10]. Although it can be seen that the method shows promising results under stationary conditions, it is not possible to use it on plants with a variable power output. Figure 2 shows the comparison between the measured and the calculated precipitation efficiency at plant 2. This plant feeds an industrial process with steam. Hence, it is controlled by the overall heat demand of the production process. As a result, there is no defined stationary operation and the calculated precipitation constant for this plant fluctuates.

It can be seen that the precipitation efficiency fluctuates. This is due to changes in the operation status of the plant. The current output of the plant cannot be assigned a numerical value, but only rough changes can be determined from the parameters on site. For example, after minute 350 , the plant is set in partial load operation. This means that the actual precipitation efficiency rises due to a lower gas velocity. This behaviour cannot be predicted with the method that had previously been proposed. It is thus impossible to calculate the precipitation efficiency using Eq. 1 for these types of operation modes.

A different approach that uses threshold values is necessary in order to find a surveillance method that can be uniformly applied to all plants.

\subsection{Using threshold values}

To find suitable threshold values for defining an efficient operation of the ESP, the operation points when the measured precipitation efficiency is above the defined target values are marked. In Fig. 3, every valid operation point is marked with a circle, meaning that the target value was reached in each case. There is no clear distinction between the measured effective and ineffective operating points. Accordingly, operating states existed at which the precipitation efficiency was sufficiently high, but this was not reflected in high current and voltage values. Possible causes could be periods with very low particulate matter levels. In these cases, the integrated cyclone and low precipitation efficiency of the electrostatic precipitator would also be sufficient to maintain the target value. At very low particulate matter concentrations, the transport of charge carriers from the spray electrode to the collecting electrode is also limited, and the measurable current would be low.

The second problem is the conditions with high voltage and current values, although the collection efficiency was lower than the target. This can happen, for example, when very high particulate matter concentrations occur during cleaning events. On the one hand, the precipitator may be overloaded, and on the other hand, the delay in particulate matter cloud detection may lead to an incorrectly calculated efficiency. This effect has already been discussed and also leads to a falsified result [10]. Similar behaviour can also be seen at the other plants. 
Fig. 2 Comparison of the calculated and measured precipitation efficiency for plant 2

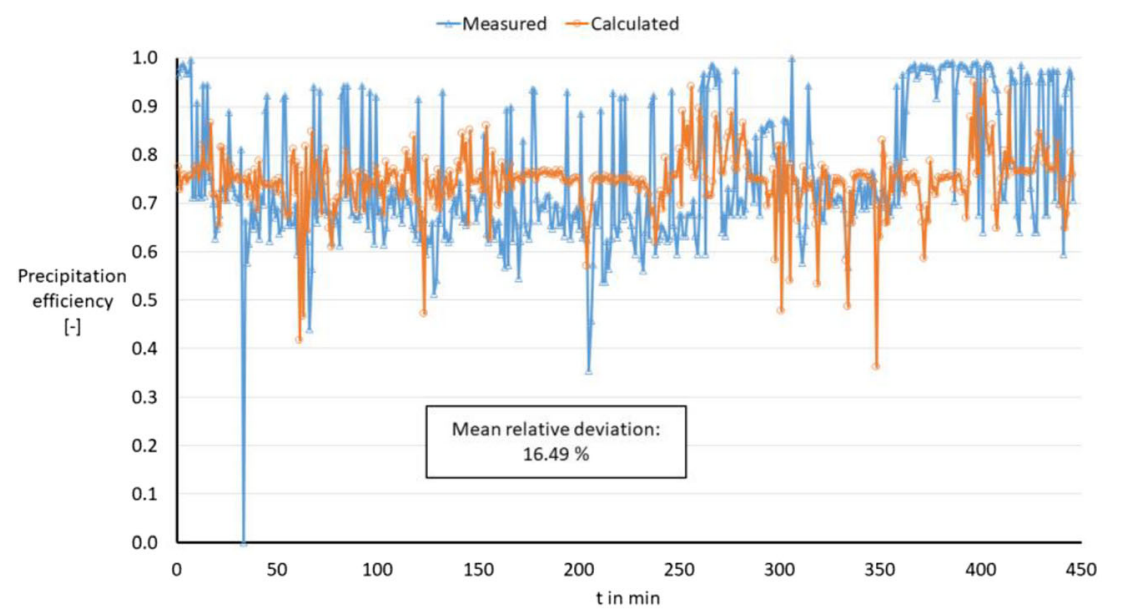

This behaviour of voltage and current can be seen at the other plants as well. There is no clear distinction between efficient and inefficient operating points either (Appendix; Figs. 11, 12, and 13).

It can be seen that the current and voltage signals do not correlate clearly with an efficient precipitation process. Individual events with very high or low particulate matter concentrations lead to operating states that are misinterpreted, both in the direction that efficient points are actually considered inefficient and vice versa. This makes it necessary to carry out a statistical evaluation aimed at permitting a fair assessment of the precipitator efficiency.

When applying the threshold values, it is evident that misinterpreted points occur. However, what is not apparent from the figures is the number of errors. Since points may overlap here, the actual number must be evaluated. This is done by varying the threshold of current and voltage and counting how many events are defined as efficient operating points - even though the measured precipitation efficiency is too low-and how many are correspondingly inefficient, even though they are efficient. Figure 4 shows the number of incorrectly interpreted operating points at plant 1 , relative to the total operating points, for the different threshold values.
As it would be expected, the occurrence of events erroneously rated as "efficient" decreases with an increased threshold value and the frequency of points erroneously rated as "inefficient" increases. This plant provides a good basis for discussing the selection of possible threshold values. If the objective is to generate as few misinterpretations as possible, the voltage threshold would be $20 \%$ of the nominal voltage and the current threshold would be $50 \%$ of the current. In this case, the discussion of the voltage threshold should also consider that the ratio of mistakes is rather constant until 0.7. This indicates that it would be beneficial to use $60 \%$ of the nominal value, because it is the highest value before a significant increase starts. This reduces the weight of possible errors in the statistical evaluation. If the aim is to achieve as similar a disadvantage as possible, the thresholds should be $70 \%$ for voltage and $60 \%$ for current. In both cases, the relative ratio of misinterpretations would be approx. $10 \%$.

In the case of plant 2 (Fig. 5) and plant 3 (Fig. 6), it can be seen that the ratio of errors increases very late when the threshold values are increased. This is due to the relatively uniform operation of the precipitators. Although this does not provide any new information about a good placement of the threshold values, it is possible to monitor both installations with the
Fig. 3 Efficient and inefficient operation points of the ESP at plant 1 evaluated based on the chosen target value

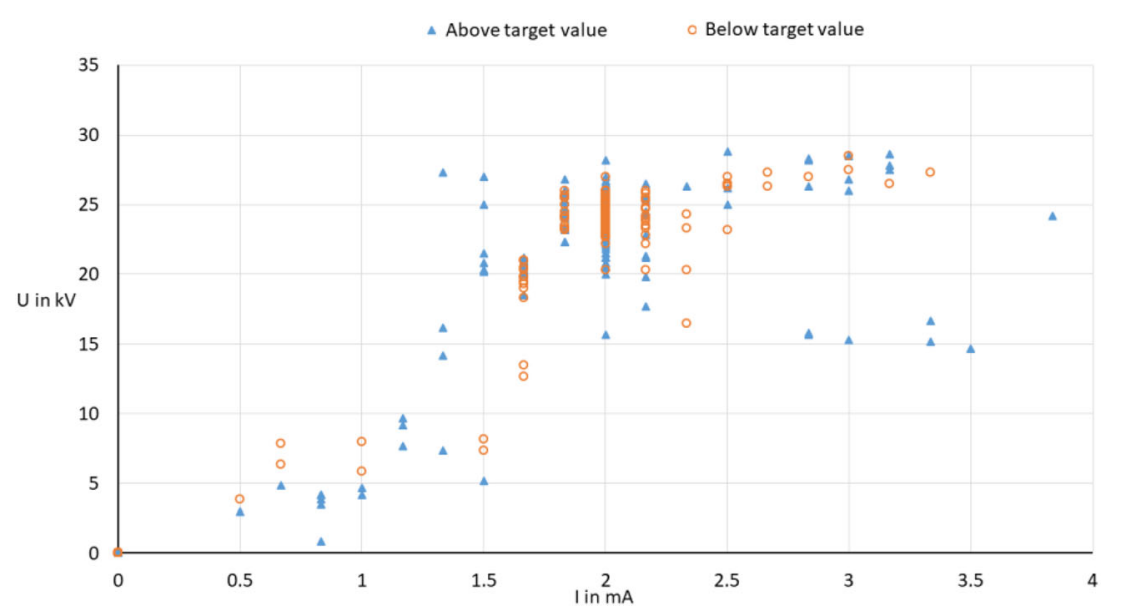


Fig. 4 Relative quantity of falsely counted events when defining a voltage and current threshold for plant 1

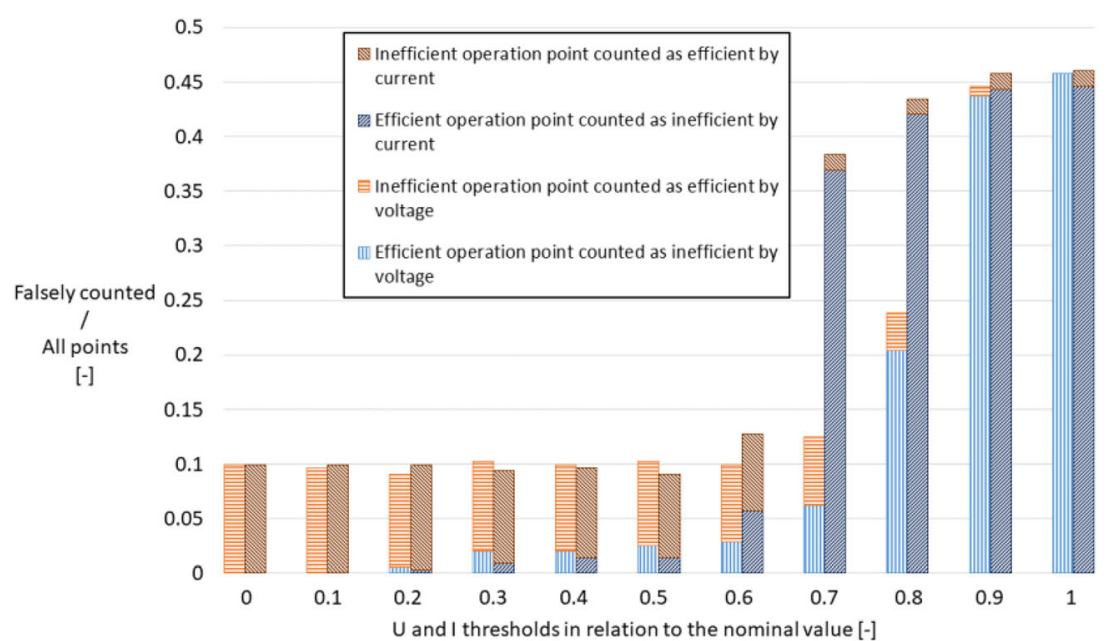

threshold values from plant 1 as well. This suggests that a uniform definition may be possible.

At plant 4 (Fig. 7), the modulating current signal helps to find a suitable threshold value. Suitable threshold values would be $70 \%$ of the nominal voltage and $50 \%$ of the nominal current, which are almost the same as those of plant 1 .

In Switzerland, the threshold values are $50 \%$ of the nominal voltage and $20 \%$ of the nominal current [11]. Table 1 shows the ratio of falsely counted events in respect to all operation points if these values are used to evaluate the operation of the four plants.

It can be seen that at plant 1 , the proportion of misinterpretations is $14.2 \%$. This is due to the modulating mode of operation of the ESP, which produces many values in the low-tomedium power range of the high-voltage generator. As discussed before, it is possible to use these threshold values for plants 2 and 3. However, due to missing values in the low and medium power range, the results do not provide further useful information in order to define suitable thresholds. At plant 4, the threshold values used in Switzerland are fitting as well. Before the legal authorities can discuss the determination

Fig. 5 Relative quantity of falsely counted events when defining a voltage and current threshold for plant 2 of the threshold values used in order to legally implement the monitoring method, it will be necessary to answer the two questions in the following section.

\subsection{Consequences for the operation of ESPs}

\subsubsection{What is the legal purpose of the precipitator?}

In a technical sense, the task of the precipitator is to reduce particulate matter emissions. Which target value is to be used to define success is not clearly defined. However, since an emission limit value is defined, compliance with this value can be defined as a target. This has the benefit that the target is also covered by the valid regulations, but it also has the disadvantage that not only the function of the precipitator is decisive for compliance with the limit value. Indeed, if the firing system is operated incorrectly, for example, because inferior fuel is used, so much particulate matter can be created that even a well-functioning precipitator will not be sufficient to comply with the limit value. This would result in a negative

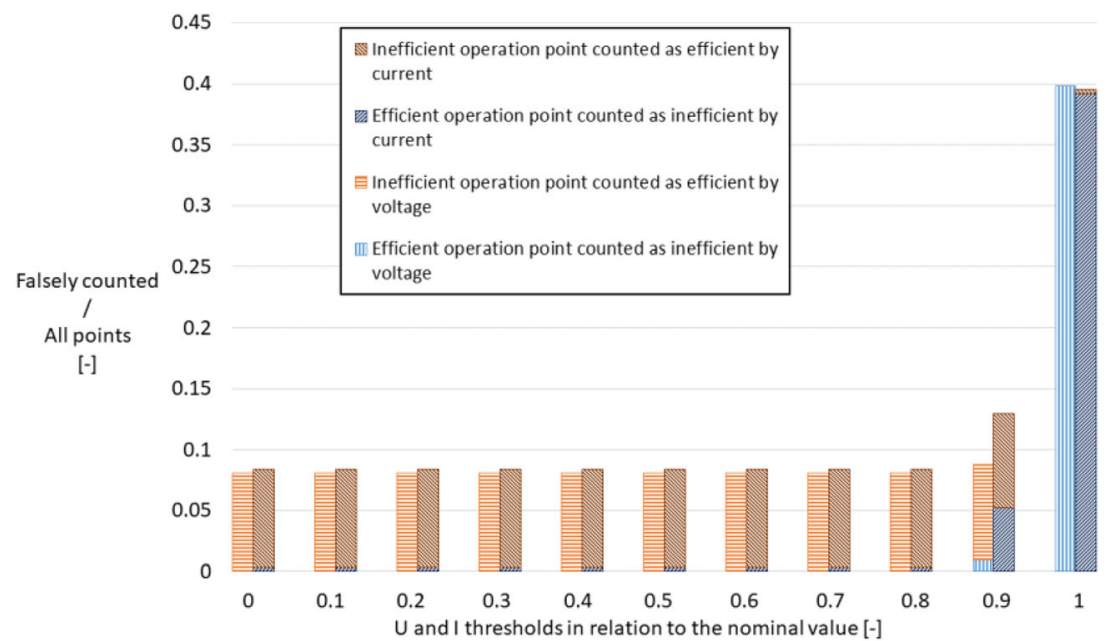


Fig. 6 Relative quantity of falsely counted events when defining a voltage and current threshold for plant 3

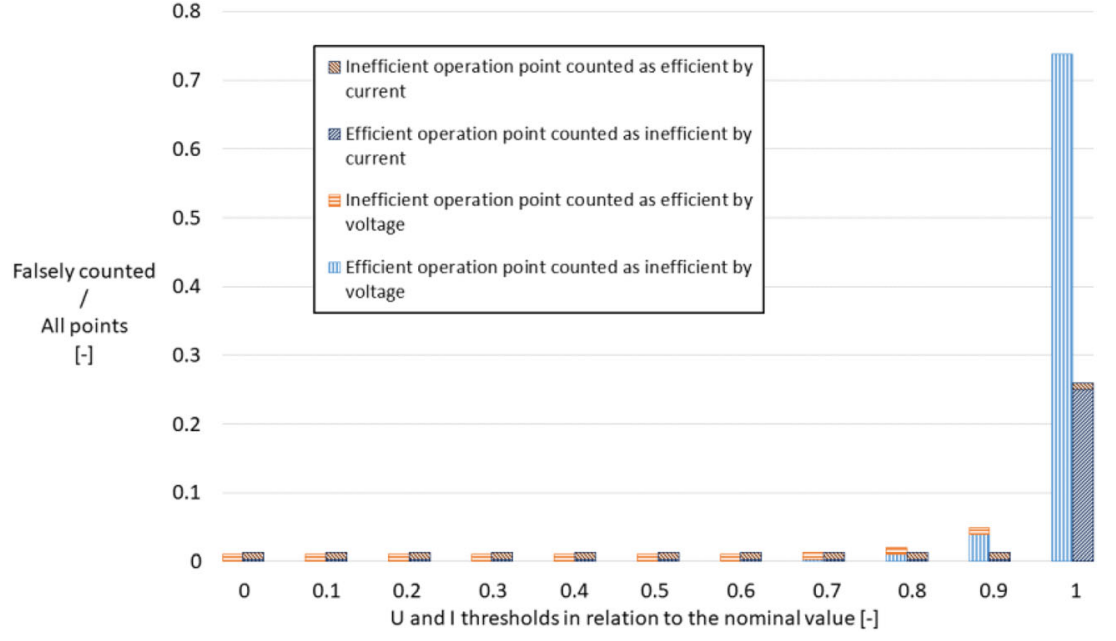

assessment of the ESP, even if other factors have to be blamed for malfunction.

The other option is to set a sufficiently high precipitation efficiency as a target value. This focuses only on the function of the ESP, but does not necessarily mean that the limit value is met. Overall, the investigations show that there are only isolated operating points at which the change in the target value influences the evaluation of the precipitator. Under normal circumstances, the precipitation efficiency also correlates very well with compliance with the limit value. Nevertheless, a target value must be defined for the implementation of legally required monitoring.

\subsubsection{Is one misinterpretation more severe than another?}

If the defined thresholds are increased, there is an increase in the frequency of the operating points, which are actually efficient but are assessed as inefficient, but there is a decrease in the frequency of the opposite misinterpretation. Thus, it makes sense to look for a minimum of total errors in between. For this purpose, however, it has to be decided whether one error is less severe than the other. If an operating point is considered efficient even though it is not, this error is disadvantageous for the legislation. Precipitator malfunctions are more likely to be accepted. However, if an operating point is wrongly judged inefficient despite being efficient, the precipitator manufacturer is at a disadvantage because their product will be judged worse. Since a complete exclusion of the respective faults is only possible with the extreme values of zero and one, it has to be decided whether a weighting of the faults is present, an overall minimum of faults is aimed at, or whether both sides should be equally disadvantaged. Without more information on the sources of misinterpretation, minimising the sum of misinterpretation should be the target.

In conclusion, the method presented can evaluate the effective operation of the ESP, but there are misinterpretations that need to be considered. Yet, the impact of these faults can be reduced if the legislation implements rules aligned with the requirements of continuous measurement in the Directive (EU) $2015 / 2193$ [31]. Therefore, the operation is valid if all monthly average values are within the limit, all daily average values are below $110 \%$ of the limit and $95 \%$ of all hourly
Fig. 7 Relative quantity of falsely counted events when defining a voltage and current threshold for plant 4

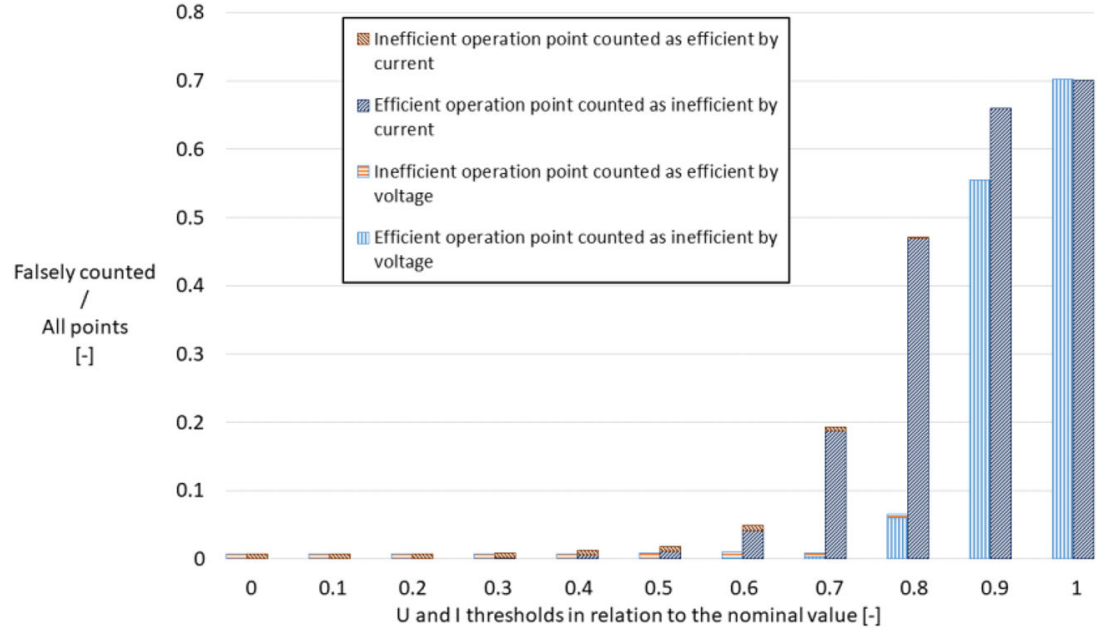


Table 1 Ratio of falsely counted events in respect to all operating points, using the threshold values of FAQ 38 in Switzerland

\begin{tabular}{cllr}
\hline Plant & Inefficient counted as efficient & Efficient counted as inefficient & Overall \\
\hline 1 & $7.7 \%$ & $6.5 \%$ & $14.2 \%$ \\
2 & $8.1 \%$ & $0.0 \%$ & $8.1 \%$ \\
3 & $1.0 \%$ & $0.0 \%$ & $1.0 \%$ \\
4 & $0.8 \%$ & $0.1 \%$ & $0.9 \%$ \\
\hline
\end{tabular}

average values are below $200 \%$ of the limit value. Applying similar rules would reduce the influence of misinterpretations.

\section{4 "Fire on" signal}

The definition of a "Fire on" signal is crucial in evaluating precipitator performance. This signal should indicate to the ESP that the combustion is running and producing particulate matter emissions. After receiving this signal, the ESP control determines if it is possible to switch on the high voltage supply and start operation. With this information, the availability or the failure times can be determined. This step is independent of the choice of method used to monitor the ESP. Even when a measurement device is chosen, a suitable "Fire on" signal will still be needed. In a previous work, it was discussed whether the flue gas temperature could be a suitable signal trigger, without the need for any signal coming from the combustion plant [10]. The definition of the temperature threshold value will be further discussed in this section. Here too, the target first needs to be defined, since the choice of threshold affects the duration and timing of the "Fire on" signal. This is illustrated in Fig. 8.

The tiled area represents the duration of the "Fire on" signal when $150{ }^{\circ} \mathrm{C}$ is used as the threshold and the dotted area accordingly for $100{ }^{\circ} \mathrm{C}$. On the one hand, it can be seen that the duration of the signal increases at a low threshold value and that short phases caused by the ember preservation state are also taken into account. A sufficiently long signal duration is positive, since less particulate matter is emitted. The "Fire on" signal should be active as long as emissions occur, since the precipitator should also be running at this time. To evaluate this, an attempt is made to determine the particulate matter emissions that would occur if the precipitators were switched off due to the missing signal. For this purpose, the measured particulate matter concentrations are summed up to the total mass of emitted matter using the measured flow velocity. Since the differential pressure sensor cannot be used at low flow velocity, a flow velocity of $1 \mathrm{~m} / \mathrm{s}$ is assumed for the phases of operation where no fans are used. This is only an estimated value, as no literature values are available and the velocity cannot be calculated on a theoretical basis. This is then considered in relation to the selected threshold value.

One further evaluation criterion is that excessive precipitator running times may lead to increased power consumption and ozone formation due to a high oxygen content in same operating phases [32]. Accordingly, the ESP should not be operated for longer than necessary.

These two opposing influences can be used to determine an optimal temperature threshold.

Figure 9 shows the progression of the two opposing effects. As the threshold increases, the required precipitator run time decreases, but the sum of emissions increases at times when the precipitator is turned off due to the missing "Fire on" signal. At the intersection of the two curves is the threshold temperature at which the sum of both effects is minimal. In this installation, this would be a temperature of $136{ }^{\circ} \mathrm{C}$. If the
Fig. 8 Visualisation of the influence of the chosen temperature threshold value on the operating time of the precipitator; example from plant 1 at full load from minute 150 to 475

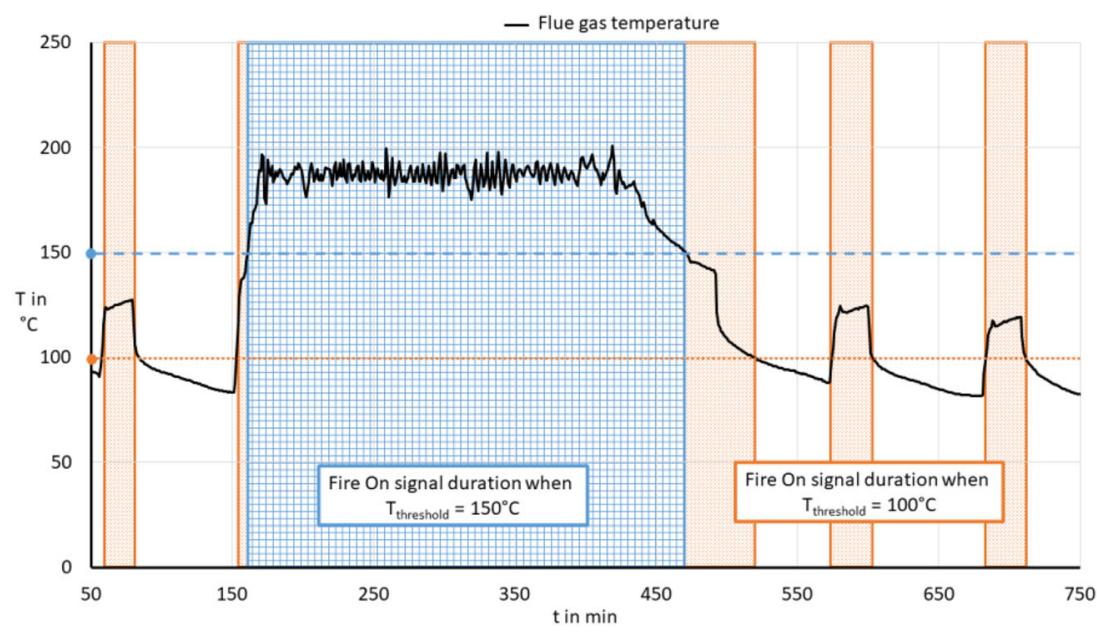


Fig. 9 Relative operating time in relation to the total monitoring time and unprecipitated emissions in relation to the chosen threshold temperature at plant 1

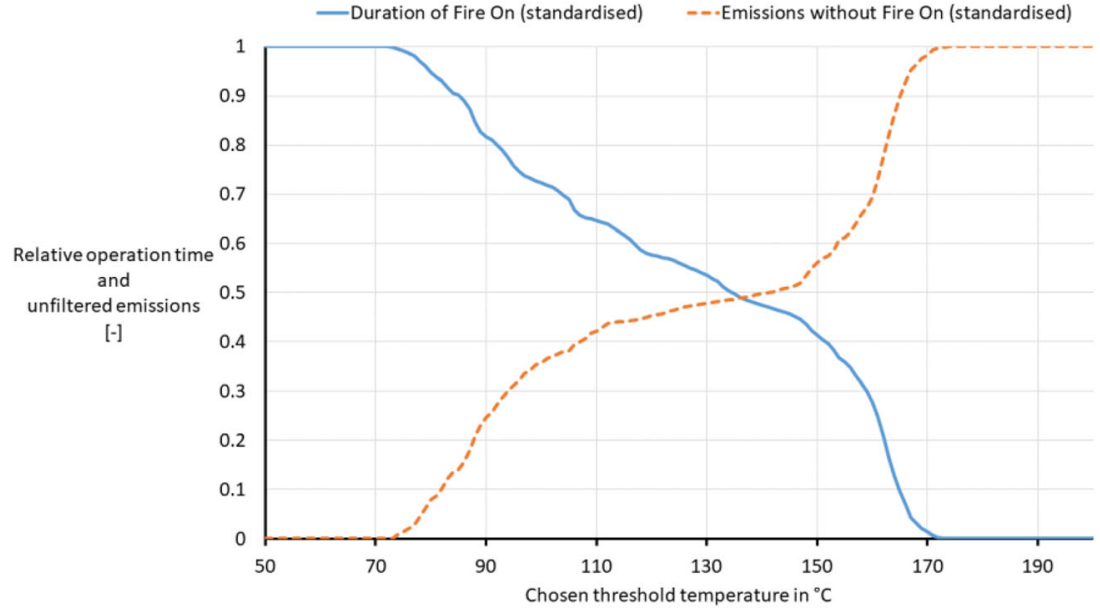

repeated for plants 2 and 4 because there were no shutdowns while the measurements were being made.

\section{Summary and conclusion}

This work follows on from the previous work by approaching the problems with plants operating with modulating heat output. The method for calculating precipitation efficiency based on the "Deutsch equation" only provides satisfactory results if the plant is operating at stationary conditions [10]. For plants with modulating power, however, the uncertainty is too high. The use of threshold values, as applied in Switzerland, is much more efficient. It was shown that when operation points were evaluated as efficient by the method, the measured precipitation efficiency could be too low and vice versa. The determination of the thresholds can therefore be measured by how many values are incorrectly interpreted as being efficient or inefficient operating points. The results indicate that suitable threshold values that are uniform for all evaluated plants can be found. Nevertheless, the legislators must decide on the actual values. To do this, the legal task of the
Fig. 10 Relative operating time in relation to the total monitoring time and unprecipitated emissions in relation to the chosen threshold temperature at plant 3

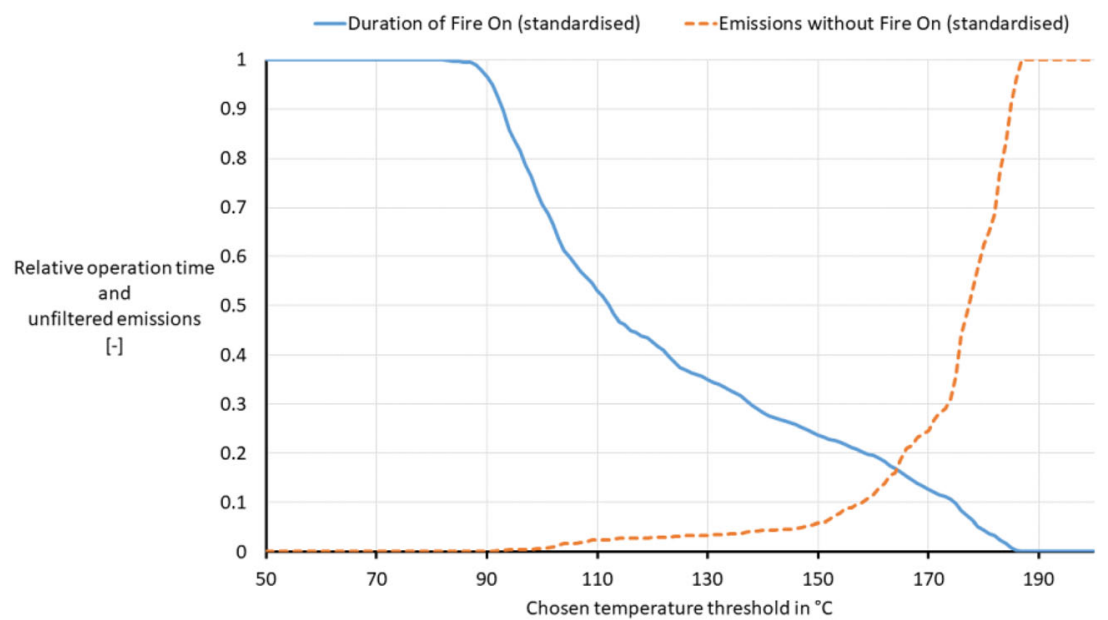


precipitator needs to be defined. Either the task is to reduce the particulate matter emissions to meet the legal requirements of the plant or it is to achieve a specific precipitation efficiency. The pros and cons of both viewpoints have been discussed. In addition, legislators need to clarify whether one kind of misinterpretation is more severe than the other.

Promising approaches are also emerging in terms of how combustion operation is defined. If the exhaust gas temperature before the ESP is used as a reference signal, the "Fire on" signal can be detected accurately and cost-effectively. However, in such a case, the actual threshold value must be individually determined for each system. Two criteria for decision-making are shown. Lowering the threshold temperature leads to longer operating times and therefore more power consumption as well as a higher risk of producing ozone. Higher values, in contrast, lead to shorter operating periods and increase the particulate matter emissions that occur because the precipitator is turned off. Here, legislators also need to clarify the optimum threshold value.

It has been shown that the use of threshold values for voltage and current for the evaluation of ESP operations, as well as the use of flue gas temperature for the definition of firing operations, allows a future continuous monitoring of an effective operation of electrostatic precipitators.

The results suggest that threshold values of $70 \%$ of the nominal voltage, $60 \%$ of the nominal current and a flue gas temperature threshold of $150^{\circ} \mathrm{C}$ are applicable to all evaluated plants. These values can be used in a uniform monitoring method.

Future investigations will focus on generating more data from different plants to support the findings. This will include the determination of the influence of the particle number distribution and the chemical composition of the particles. Additionally, it will be evaluated whether the results can be transferred to smaller ESPs.

Acknowledgements The authors would like to thank the Fachagentur Nachwachsende Rohstoffe (FNR) and the German Federal Ministry of Food and Agriculture for funding and supporting this project.

Funding Open Access funding enabled and organized by Projekt DEAL. This study was supported by the Fachagentur Nachwachsende Rohstoffe (FNR) and the German Federal Ministry of Food and Agriculture with the grant number 22000617.

GFNR

\section{Compliance with ethical standards}

Conflict of interest The authors declare that they have no conflict of interest.

Abbreviations ESP, electrostatic precipitators; MCP, medium combustion plants; I, current; U, voltage; FNR, "Fachagentur Nachwachsende Rohstoffe"; BImSchV, "Bundesimmissionsschutzverordnung", Federal Immission Control Act; $\eta$, precipitation efficiency; $k$, precipitator constant; MW, megawatt; min, minutes; $\mathrm{mg} / \mathrm{m}^{-3}$, milligrammes per cubic metre; $\mathrm{kV}$, kilovolt; $\mathrm{mA}$, milliampere; ${ }^{\circ} \mathrm{C}$, degrees Celsius

\section{Appendix}

Fig. 11 Efficient and inefficient operation points of the ESP at plant 2 evaluated based on the chosen target value

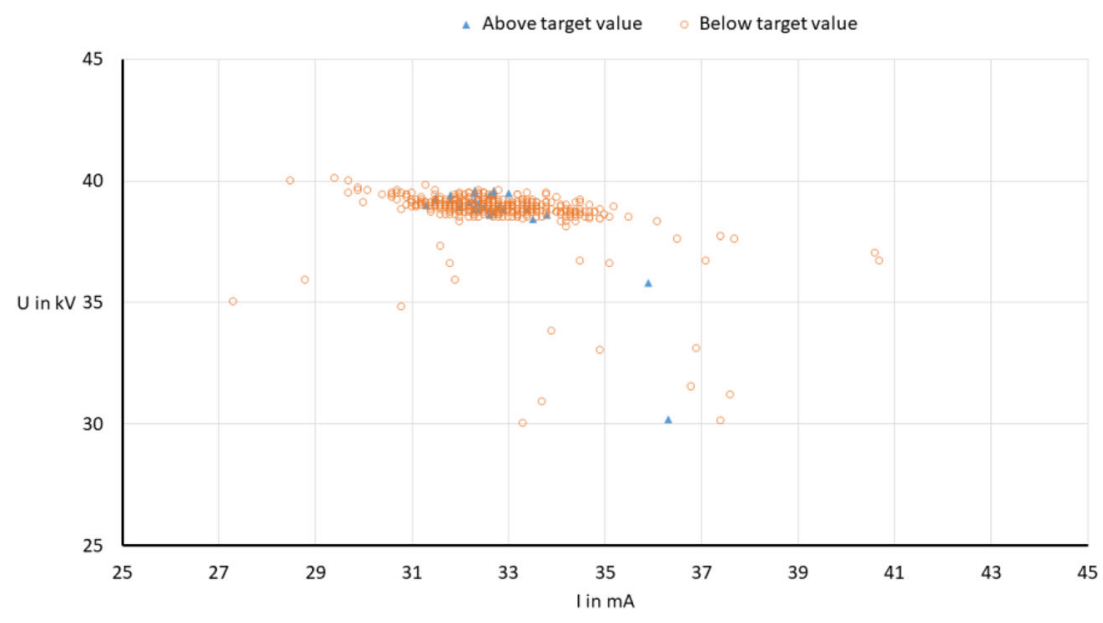


Fig. 12 Efficient and inefficient operation points of the ESP at plant 3 evaluated based on the chosen target value
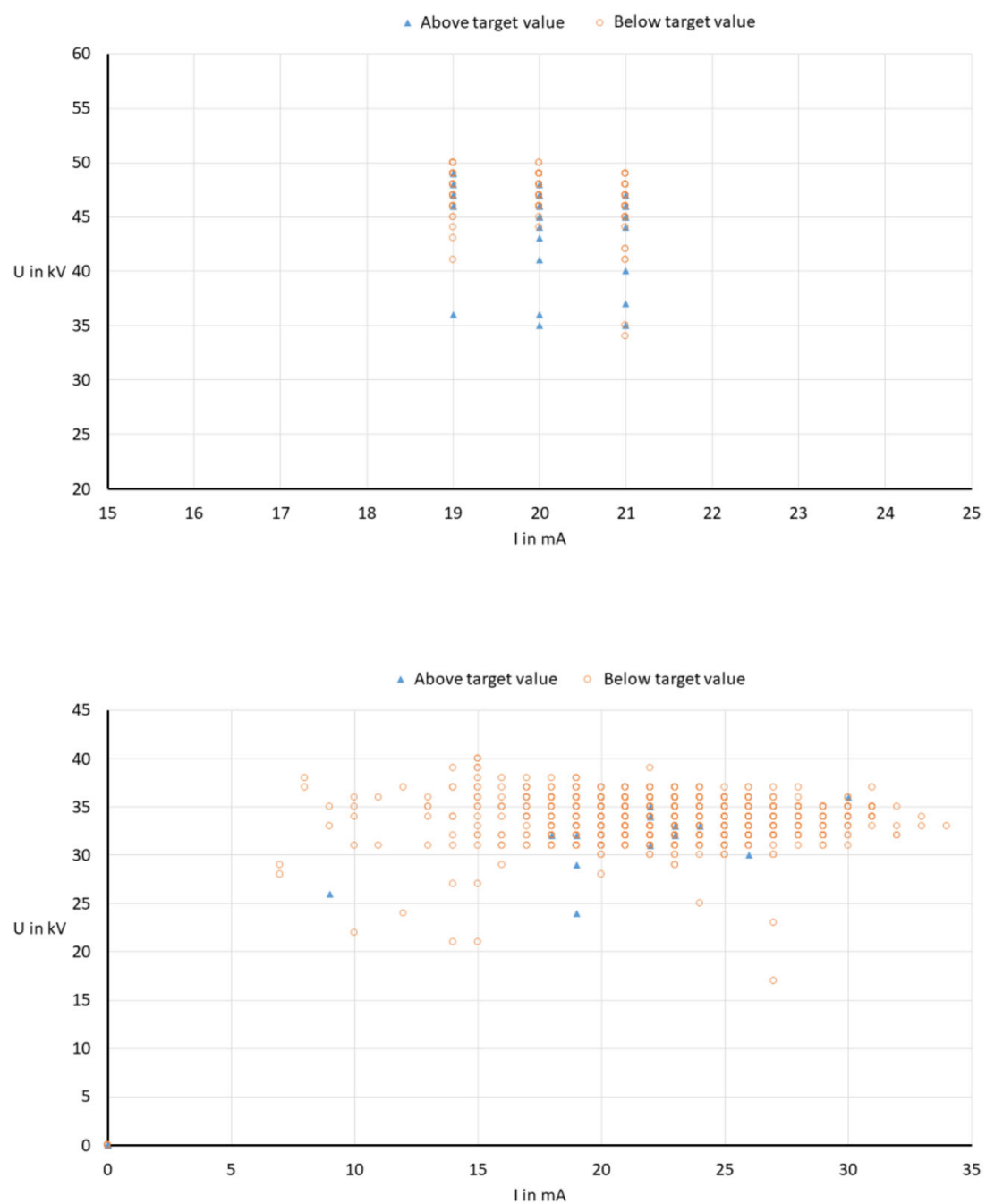

Fig. 13 Efficient and inefficient operation points of the ESP at plant 4 evaluated based on the chosen target value
Open Access This article is licensed under a Creative Commons Attribution 4.0 International License, which permits use, sharing, adaptation, distribution and reproduction in any medium or format, as long as you give appropriate credit to the original author(s) and the source, provide a link to the Creative Commons licence, and indicate if changes were made. The images or other third party material in this article are included in the article's Creative Commons licence, unless indicated otherwise in a credit line to the material. If material is not included in the article's Creative Commons licence and your intended use is not permitted by statutory regulation or exceeds the permitted use, you will need to obtain permission directly from the copyright holder. To view a copy of this licence, visit http://creativecommons.org/licenses/by/4.0/.

\section{References}

1. Hoegh-Guldberg O, Jacob D, Taylor M, Bindi M, Brown S, Camilloni I, Diedhiou A, Djalante R, Ebi KL, Engelbrecht F, Guiot J, Hijioka Y, Mehrotra S, Payne A, Seneviratne SI, Thomas A, Warren R, Zho G (2018) Impacts of $1.5^{\circ} \mathrm{C}$ global warming on natural and human systems. In: Masson-Delmotte V, Zhai P, Pörtner H-O, Roberts D, Skea J, Shukla PR, Pirani A, Moufouma-Okia W, Péan C, Pidcock R, Connors S, Matthews
JBR, Chen Y, Zhou X, Gomis MI, Lonnoy E, Maycock T, Tignor M, Waterfield T (eds) Global Warming of $1.5^{\circ} \mathrm{C}$. An IPCC Special Report on the impacts of global warming of $1.5^{\circ} \mathrm{C}$ above pre-industrial levels and related global greenhouse gas emission pathways, in the context of strengthening the global response to the threat of climate change, sustainable development, and efforts to eradicate poverty

2. United Nations Report Of The Secretary-General On The 2019 Climate Action Summit And The Way Forward In, p 2020

3. Edenhofer O (ed) (2012) Renewable energy sources and climate change mitigation: special report of the intergovernmental panel on climate change. Cambridge University Press, Cambridge

4. Kaltschmitt M, Hartmann H, Hofbauer H (2016) Energie aus Biomasse. Berlin, Heidelberg, Springer Berlin Heidelberg

5. Nyström R, Lindgren R, Avagyan R, Westerholm R, Lundstedt S, Boman C (2017) Influence of wood species and burning conditions on particle emission characteristics in a residential wood stove. Energy Fuel 31(5):5514-5524. https://doi.org/10.1021/acs. energyfuels.6b02751

6. McLean KJ (1988) Electrostatic precipitators. IEE Proc. A Phys. Sci. Meas. Instrum. Manage. Educ. Rev. UK 135(6):347. https:// doi.org/10.1049/ip-a-1.1988.0056

7. von Stackelberg J, Schmoch M (2018) Handbuch Elektrofilter: Physik, Hochspannungsversorgung, Erdung und Auslegung. Vieweg, Wiesbaden 
8. (2019) Vierundvierzigste Verordnung zur Durchführung des Bundes-Immissionsschutzgesetzes: 44. BImSchV

9. Rösch C, Kaltschmitt M (1999) Energy from biomass - do nontechnical barriers prevent an increased use? Biomass Bioenergy 16(5):347-356

10. Alt B, Klüh D, Gaderer M (2019) Development of an online monitoring method for electrostatic precipitators on commercial biomass combustion plants. Biomass Conv. Bioref 476(1):43 EP. https://doi.org/10.1007/s13399-019-00579-y

11. QM Holzheizwerke Holzenergie Schweiz, FAQ38, 2016

12. Nussbaumer T, Lauber A (2016) Monitoring the availability of electrostatic precipitators (ESP) in automated biomass combustion plants. Biomass Bioenergy 89:24-30. https://doi.org/10.1016/j. biombioe.2016.02.027

13. Fraunhofer UMSICHT (2019)"Verbundvorhaben: FRESBI: »Optimierung der FRaktionsabscheidegrade Elektrostatischer Staubabscheider beim Einsatz in BIomassefeuerungen«: Schlussbericht"

14. Obernberger I (1997) Nutzung fester Biomasse in Verbrennungsanlagen unter besonderer Berücksichtigung des Verhaltens aschebildender Elemente, 2nd edn. dbv-Verl, Graz

15. Qi Z, Li J, Wu D, Xie W, Li X, Liu C (2017) Particulate matter emission characteristics and removal efficiencies of a low-low temperature electrostatic precipitator. Energy Fuel 31(2):1741-1746. https://doi.org/10.1021/acs.energyfuels.6b02692

16. Jaworek A, Marchewicz A, Sobczyk AT, Krupa A, Czech T (2018) Two-stage electrostatic precipitators for the reduction of PM2.5 particle emission. Prog Energy Combust Sci 67:206-233. https:// doi.org/10.1016/j.pecs.2018.03.003

17. Lübbert C (2011) Zur Charakterisierung des gequenchten Zustandes im Elektroabscheider: Dissertation: Brandenburgische Technische Universität Cottbus

18. Mizuno A (2000) Electrostatic precipitation. IEEE Trans Dielect Electr Insul 7(5):615-624. https://doi.org/10.1109/94.879357

19. Ruttanachot C, Tirawanichakul Y, Tekasakul P (2011) Application of electrostatic precipitator in collection of smoke aerosol particles from wood combustion. Aerosol Air Qual Res 11(1):90-98. https:// doi.org/10.4209/aaqr.2010.08.0068
20. Zhao Zhibin ZG (1992) New model of electrostatic precipitation efficiency accounting for turbulent mixing. J Aerosol Sci 2:115121

21. Guo B-Y, Yu A-B, Guo J (2014) Numerical modeling of electrostatic precipitation: effect of gas temperature. J Aerosol Sci 77:102115. https://doi.org/10.1016/j.jaerosci.2014.07.009

22. Skodras G, Kaldis SP, Sofialidis D, Faltsi O, Grammelis P, Sakellaropoulos GP (2006) Particulate removal via electrostatic precipitators - CFD simulation. Fuel Process Technol 87(7): 623-631. https://doi.org/10.1016/j.fuproc.2006.01.012

23. Anagnostopoulos J, Bergeles G (2002) Corona discharge simulation in wire-duct electrostatic precipitator. J Electrost 54(2):129 147. https://doi.org/10.1016/S0304-3886(01)00172-3

24. Deutsch W (1922) Bewegung und Ladung der Elektrizitätsträger im Zylinderkondensator. Annalen der Physik (373):335-344

25. White H (1963) Industrial Electrostatic Precipitation. AddisonWesley

26. Matts S, Öhnfeldt PO Efficient gas cleaning with SF electrostatic precipitators. Fläkten 163:93-110

27. Cooperman G (1984) "A unified efficiency theory for electrostatic precipitators,” 2, p. 277-285, 1984. [Online]. Available: https:// www.https://www.sciencedirect.com/science/article/abs/pii/ 000469818490101X

28. Particulate matter measurement - Dust measurement in flowing gases - Gravimetric determination of dust load, VDI 2066 Blatt 1, 2008

29. Schmitt C Manual MGAprime: 2019. MRU GmbH

30. SICK Engineering GmbH, Dusthunter SP30: Manual. 2019

31. P. Office, DIRECTIVE (EU) 2015/2193 OF THE EUROPEAN PARLIAMENT AND OF THE COUNCIL- of 25 November 2015 - on the limitation of emissions of certain pollutants into the air from medium combustion plants

32. Lebedynskyy S (2013) Energieeffiziente Abscheidung von hochkonzentrierten flüssigen Aerosolen mit einem Autogenen Raumladungsgetriebenen Abscheider: Dissertation. CottbusSenftenberg

Publisher's Note Springer Nature remains neutral with regard to jurisdictional claims in published maps and institutional affiliations. 